\title{
Does chronotherapy for essential hypertension matter by class? A systematic review and meta-analysis
}

\author{
Hongyu Kuang ${ }^{1}$ and Yunlin Chen ${ }^{1}$ \\ ${ }^{1}$ Affiliation not available
}

April 4, 2021

\begin{abstract}
Objectives The study was performed to evaluate the efficacy and safety of chronotherapy of hypertension with different medications monotherapy or a combination compared with traditional regimens Methods Three databases including PubMed, EMBASE and the Cochrane Library were searched, from the inception of each database to 10 April 2020. The Review Manager 5.4 was adopted for meta-analyses and subgroup analyses. The blood pressure delta $(\Delta)$ was used as mean of differences (MD) with $95 \%$ confidence intervals (CIs), and the estimated effect for events estimates the 95\% CIs for frequency of events. The adults with essential hypertension were treated with chronotherapy and traditional regimens. Results Twenty-eight RCTs, recruiting 1865 patients in bedtime/evening dosing and 1867 in awakening/morning dosing, were enrolled in this quantitative review. Meta-analysis showed no significant differences for overall drug-related AEs $(\mathrm{RR}=0.81, \mathrm{P}=0.17$; $\mathrm{I} 2=41 \%)$, but an obvious reduction of risk for overall withdrawals $(\mathrm{RR}=0.52, \mathrm{P}=0.005 ; \mathrm{I} 2=0.0 \%$ ) with bedtime dosing. No statistically significant differences were noted for clinic BP and diurnal BP, but 24-hour (48-hour) BP, nocturnal BP, morning BP, and non-dippers (\%) showed obvious reductions, statistically. By class, there existed different efficacy between 2 administrations, with great decrease in nocturnal BP control and changes in circadian rhythm with RAAS blockers monotherapy, but an all-day control of BP for CCBs and diuretics. With regard to a combination, no significant differences in BP management were detected and the data about beta-receptor blockers were limited. Conclusions The safety and efficacy of chronotherapy in antihypertensive drugs might be based on the classes.
\end{abstract}

\section{Hosted file}

manuscript.pdf available at https://authorea.com/users/405719/articles/516597-doeschronotherapy-for-essential-hypertension-matter-by-class-a-systematic-review-and-metaanalysis

\section{Hosted file}

Table 1 Included studies.pdf available at https://authorea.com/users/405719/articles/516597does-chronotherapy-for-essential-hypertension-matter-by-class-a-systematic-review-andmeta-analysis

\section{Hosted file}

Table 2 (safety profiles).pdf available at https://authorea.com/users/405719/articles/516597does-chronotherapy-for-essential-hypertension-matter-by-class-a-systematic-review-andmeta-analysis 


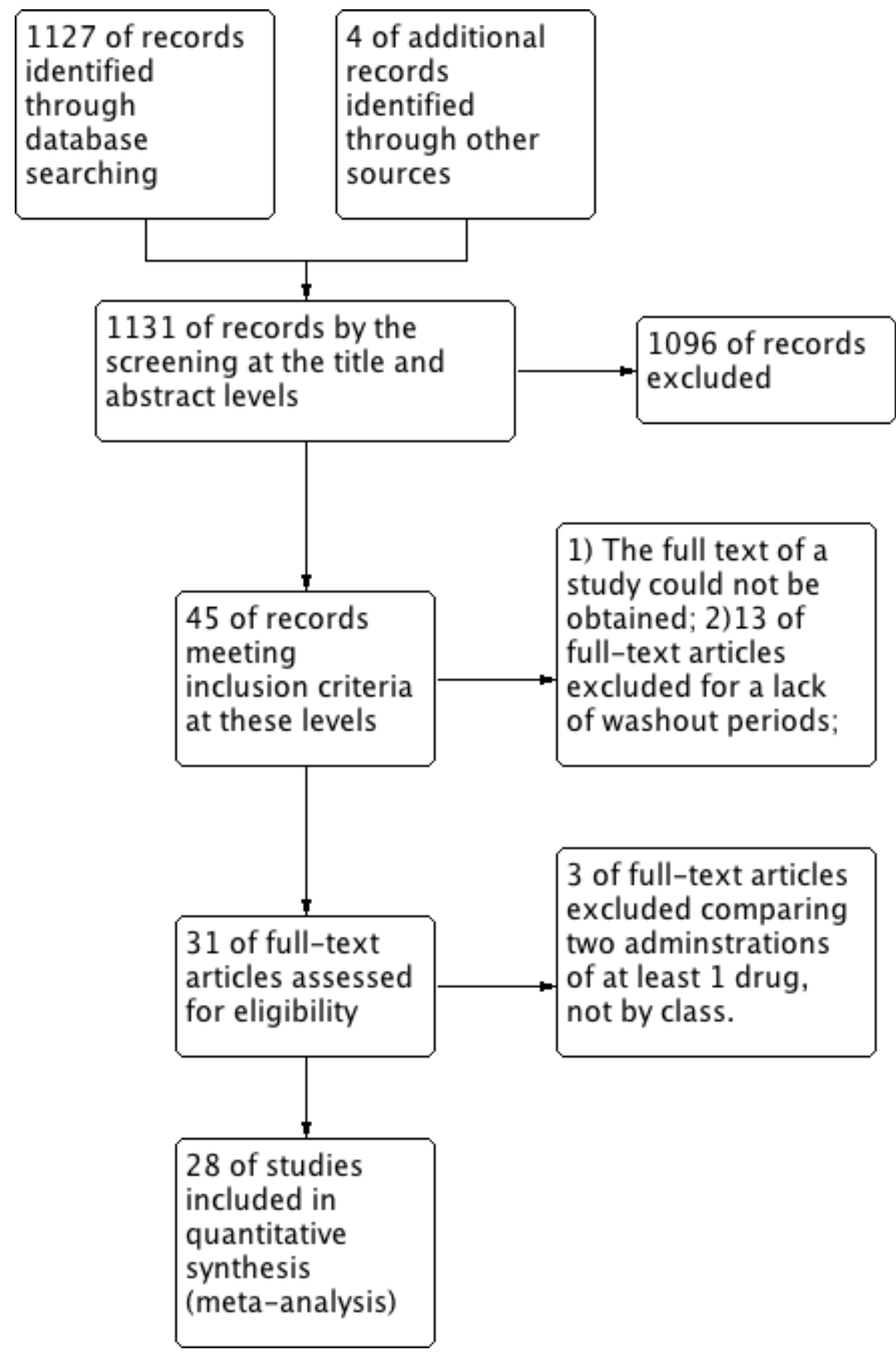




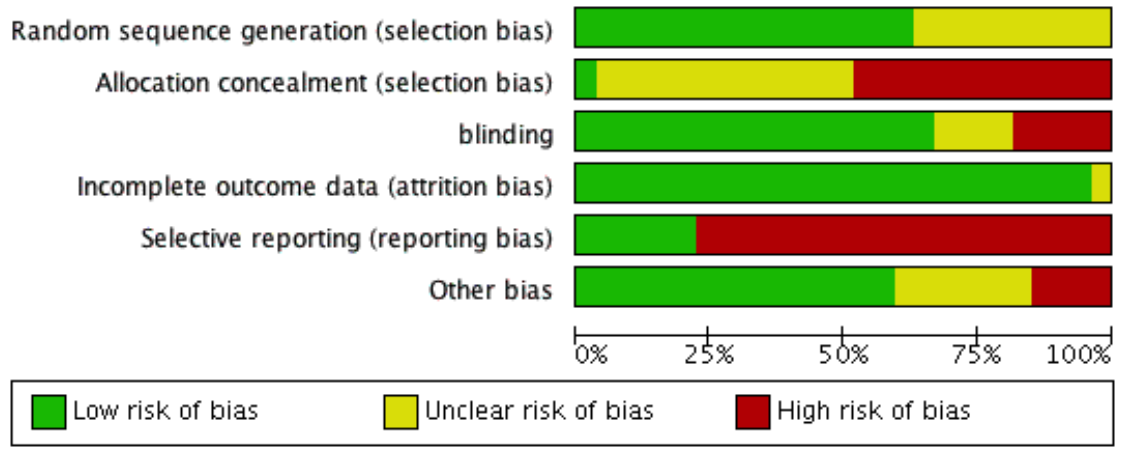




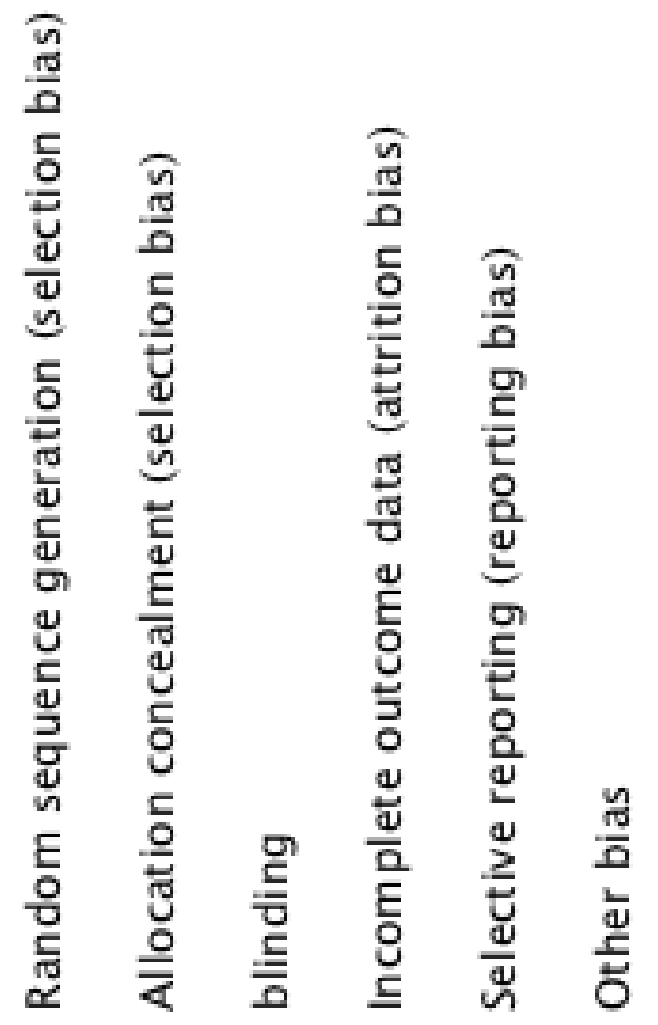

\begin{tabular}{|c|c|c|c|c|c|c|}
\hline Acelajado 2012 & $?$ & $?$ & 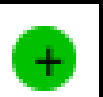 & + & & $?$ \\
\hline Asmar 2011 & $?$ & $?$ & $\odot$ & 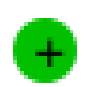 & & $?$ \\
\hline Calwo 2006 & + & & + & + & & + \\
\hline Hermida 2003 & + & & + & $?$ & & 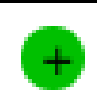 \\
\hline Hermida 2005 a & + & & $\odot$ & $\hookrightarrow$ & & 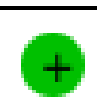 \\
\hline Hermida 2005 b & + & & $\odot$ & 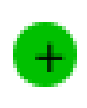 & & 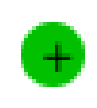 \\
\hline Hermida 2007 & + & & 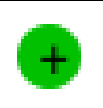 & + & & † \\
\hline Hermida 2007a & + & & 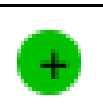 & $\dashv$ & & 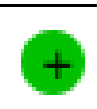 \\
\hline Hermida 2008 & + & & 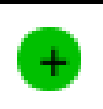 & + & & + \\
\hline Hermida $2008 \mathrm{a}$ & + & - & $\hookrightarrow$ & 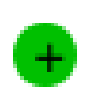 & & $\odot$ \\
\hline Hermida 2009 & + & + & $?$ & + & + & + \\
\hline ermida $2009 \mathrm{a}$ & $\odot$ & $?$ & $?$ & 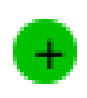 & 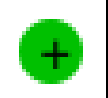 & 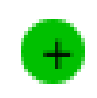 \\
\hline
\end{tabular}



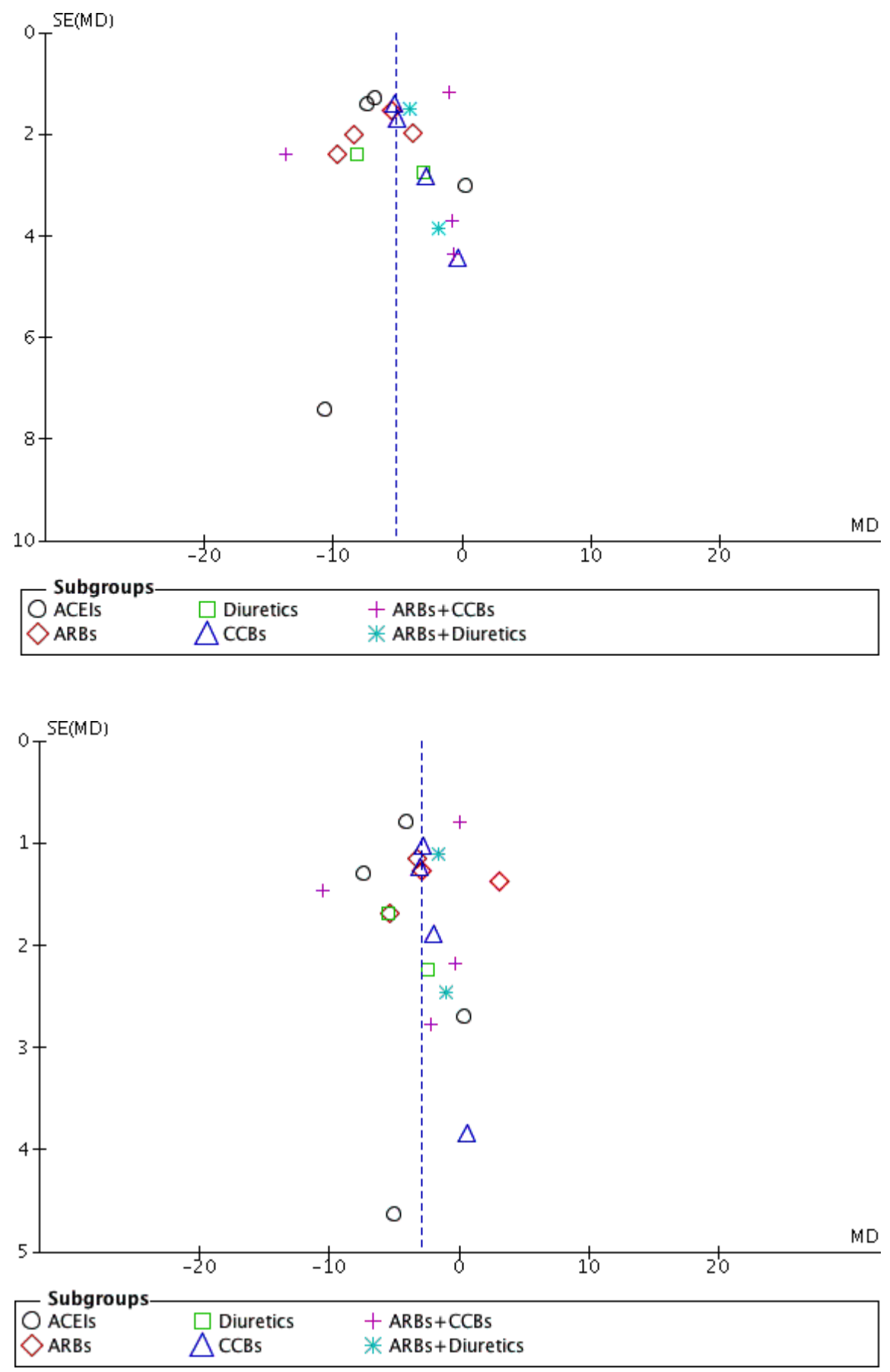


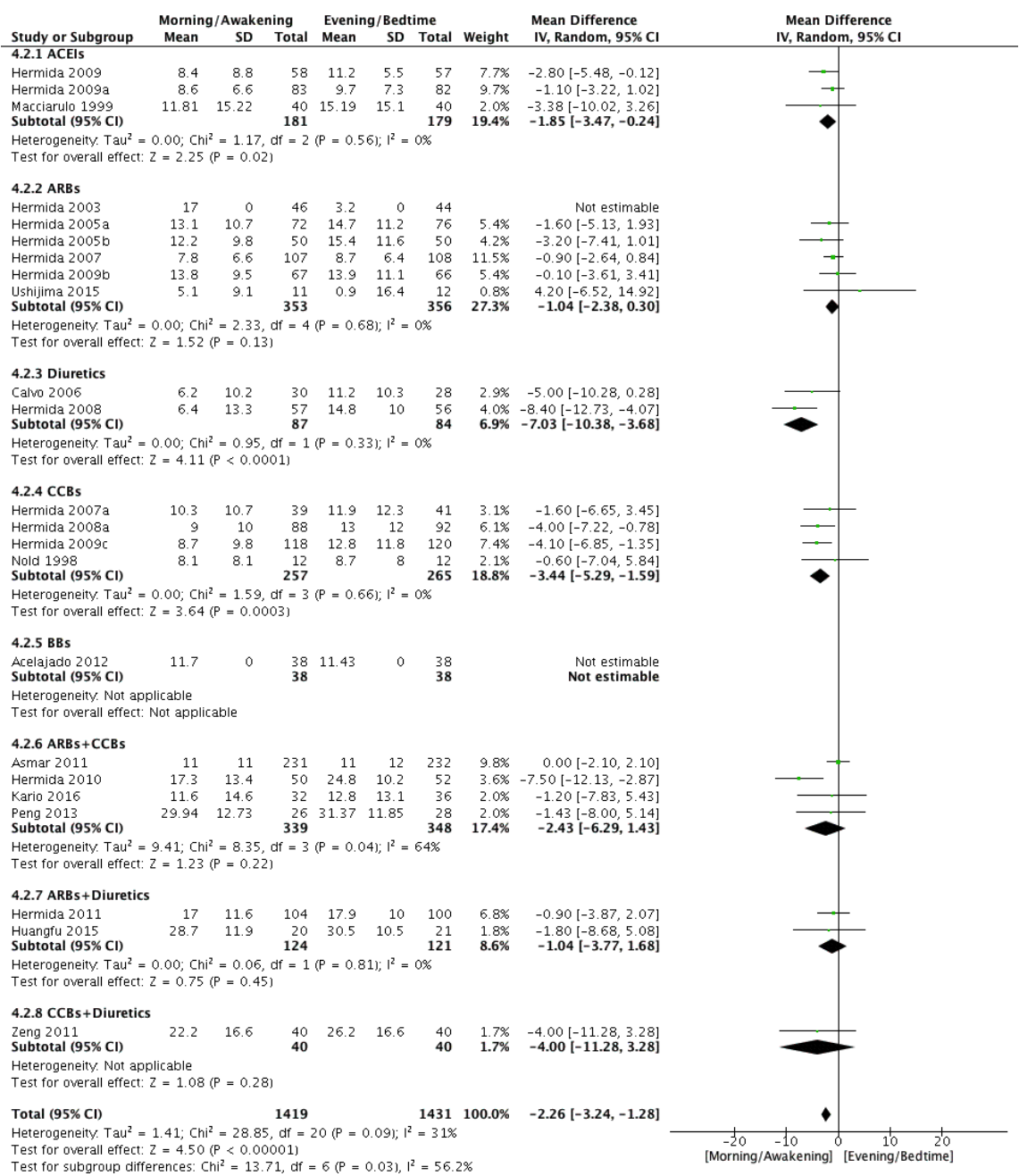




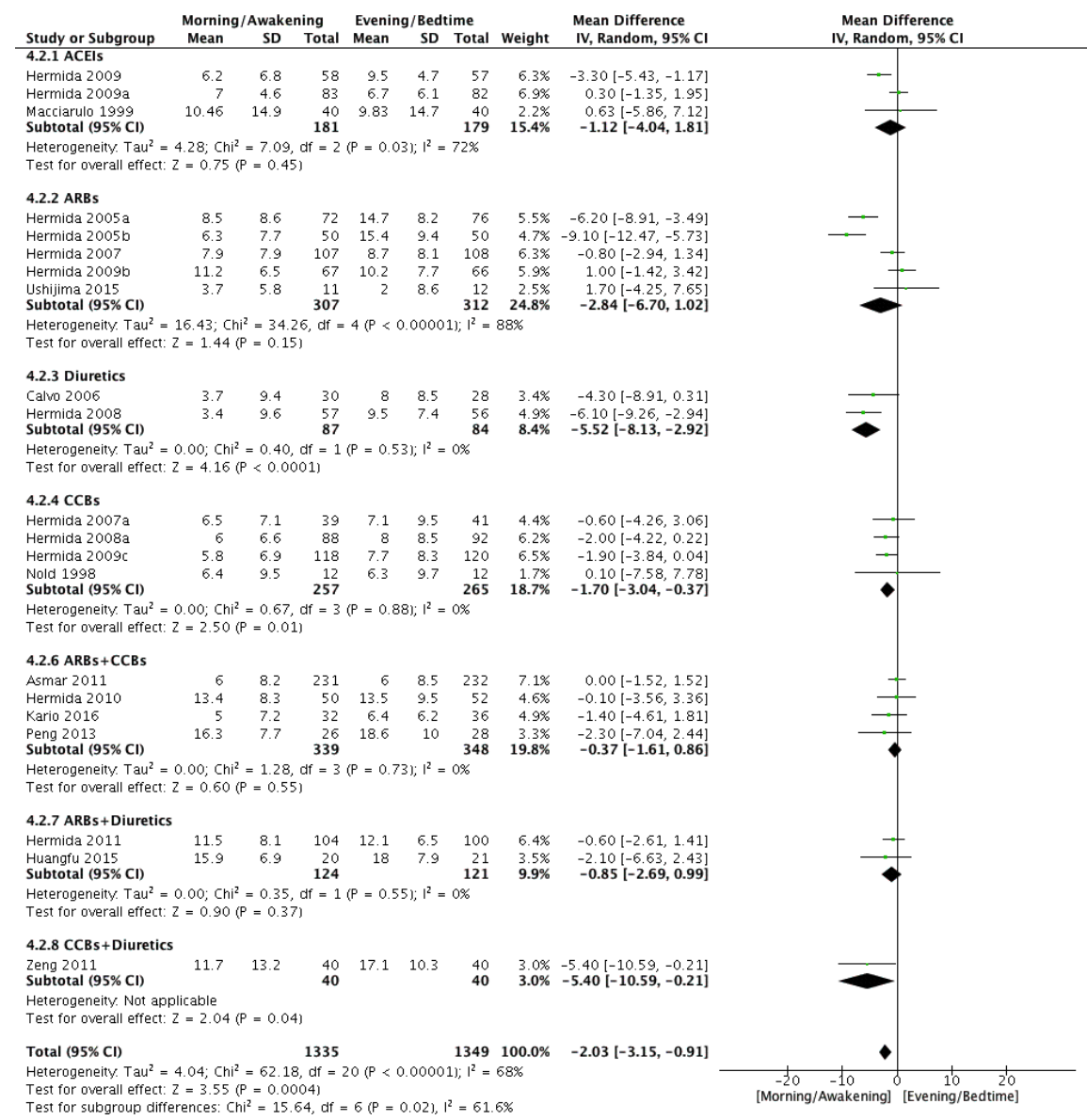

